\title{
Coloring Quasicrystals with Prescribed Symmetries and Frequencies*
}

\author{
A. El Kacimi Alaoui ${ }^{1}$ and R. Parthasarathy ${ }^{2}$ \\ ${ }^{1}$ LAMATH, Université de Valenciennes, \\ Le Mont Houy, 59304 Valenciennes Cedex, France \\ elkacimi@univ-valenciennes.fr \\ ${ }^{2}$ School of Mathematics, Tata Institute of Fundamental Research, \\ Homi Bhabha Road, Colaba, Bombay 400 005, India \\ sarathy@math.tifr.res.in
}

\begin{abstract}
We show how to color the tiles in a heirarchical tiling system so that the resulting system is not only repetitive (i.e., has the local isomorphism property) but has prescribed color symmetries as well.
\end{abstract}

\section{Introduction}

Let $T$ be a repetitive tiling of $\mathbb{R}^{n}$ by tiles congruent to a finite number of tile types. Here "repetitive" means that the tiling has "local-isomorphism" property in the following sense: given any patch of $T$ occurring in any bounded region of $\mathbb{R}^{n}$, it is repeated in any other sufficiently large region. Many interesting examples of tilings are "heirarchical," in the following sense: there are precise rules using which one can pass from any given tiling $T_{0}$ by tiles congruent to the given tile types (and matching rules ...) to a tiling $T_{1}$ with the same set of tiles on a larger scale $\tau$, where $\tau$ is a positive real number greater than one. Furthermore, when the second tiling is superimposed on the first one, the tiles of the new tiling are unions of tiles of the older tiling. Moreover, two congruent tiles of $T_{1}$ are decomposed into tiles of $T_{0}$ in exactly the same way. This second tiling $T_{1}$ gives rise to a tiling $T_{2}$ whose tiles are similar to those of $T_{0}$ in the ratio $\tau^{2}$ and so on ....

\footnotetext{
* The second author is grateful to the URA au CNRS GAT, U.F.R. de Mathématiques, Université des Sciences et Technologies de Lille for their hospitality during his stay when this work was done.
} 
In this article we consider the problem of coloring the tiles of a given heirarchical and repetitive tiling which exhibits local permutation isomorphisms (definitions below) and moreover color isolation and frequencies when these are prescribed.

Definition 1 (Local $(G, \sigma, M)$-Symmetry). Let $G$ be a finite group acting on a finite set $M: \sigma: G \times M \rightarrow M$ (the elements of $M$ will be the colors). Assume that the tiles in $T$ are colored with the elements of $M$. We say that the coloring of $T$ has local $(G, \sigma, M)$ symmetry (or simply that $T$ has $(G, \sigma, M)$-symmetry) if given $g \in G$ and given a patch $\Sigma$ of the tiles of $T$ in any bounded region, there exists $R>0$ such that in any disk of radius $R$ one can find a copy $\Sigma^{\prime}$ of the patch $\Sigma$, the only change being that the coloring of $\Sigma^{\prime}$ is obtained from that of $\Sigma$ on applying the permutation $\sigma(g)$.

Our first result is the following:

Theorem 2. Let $T$ be a heirarchical tiling which has the local isomorphism property. Then for any $(G, \sigma, M)$ there always exists a coloring of $T$ with local $(G, \sigma, M)$ symmetry.

It should be pointed out that it suffices to prove the result taking $G$ to be the full group of permutations of $M$. However, subgroups $G$ of $\operatorname{Aut}(M)$ offer more interesting possibilities as we can demand color isolation and frequencies, to be explained presently.

We find it useful to assign an element of a finite group $H$ to the relative position of a tile in a chosen higher level of the heirarchy and think of it as some sort of an "orientation." This is quite formal and is not motivated by any physical considerations. This is our definition of "relative $H$-orientation" (Definition 4). The absolute or "global $H$-orientation" (Definition 5) is the cumulative effect of applying successive relative $H$-orientations following through higher and higher levels of the heirarchy. The group $G$ which acts as the permutation of the colors may be unrelated to the group $H$ involved in the formal notion of the " $H$-orientation" of tiles. We can then ask for colorings of $T$ with $(G, \sigma, M)$-symmetry in which:

(i) ("color isolation") tiles in global $H$-orientation $h$ have to be colored only from specified $G$-stable subsets $M_{h}$ of $M$;

(ii) ("frequency") among the tiles in global $H$-orientation $h$ the distribution of tiles in color $x$ to those in color $y$ is in a given ratio which depends on the $G$-orbits of $x$ and $y$.

(The formal definitions are given elsewhere in what follows.)

Our results assert the existence of such colorings (see Theorem 10).

\section{Terminology and Results}

For simplicity, we illustrate the method of coloring in the case of tilings of $\mathbb{R}^{2}$ with the Robinson-Penrose tilings. The reader can see that they easily generalize. We begin by recalling to the reader the construction of the said tilings briefly following [GS]. 

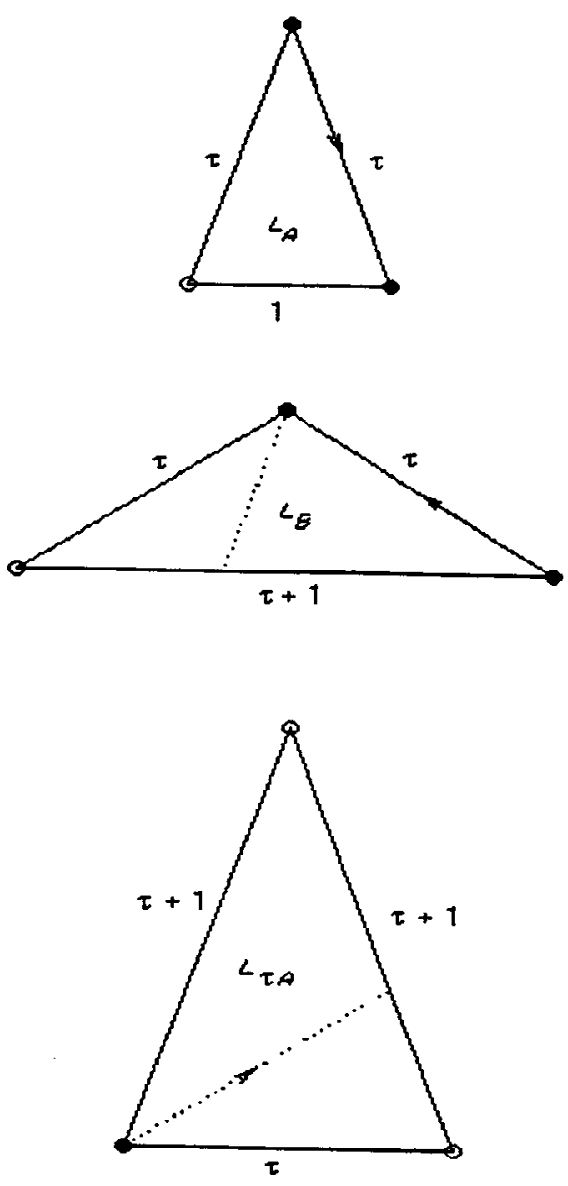
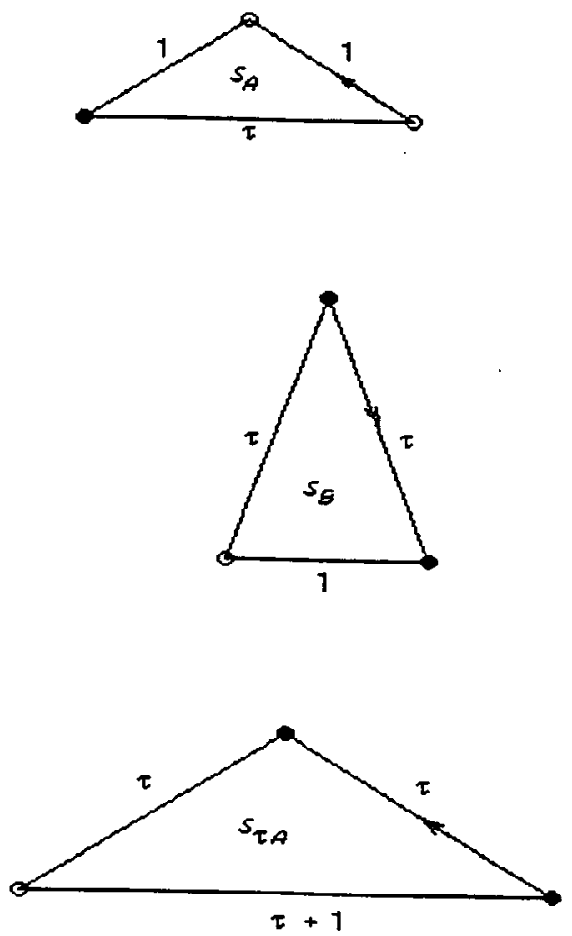

Fig. 1

\subsection{Robinson and Penrose Tilings}

One knows that by a simple technique of cutting and gluing one can pass from Robinson tilings to Penrose tilings and vice versa. We recall the basic facts about Robinson tilings as described on p. 540 of [GS]. One first considers two triangular prototiles $L_{A}$ and $S_{A}$ as represented in Fig. 1.

Both prototiles have black as well as white vertices and in both prototiles edges whose vertices have the same color are directed. (In the case of $L_{A}$ the directed edge has two black vertices while for $S_{A}$ the directed edge joins two white vertices.)

A tiling of type $A$ of the plane is defined to be one for which the tiles are isometric to either $L_{A}$ or $S_{A}$ and in which colors of common vertices and the direction of common directed edges are the same (see [GS] for a proof of the existence of such tilings). 
In a tiling $T_{0}$ of type $A$ one never finds two $S_{A}$-triangles with a common undirected short edge. In other words, on the other side of an undirected short edge of an $S_{A}$-triangle one always finds an $L_{A}$-triangle (of course with the colors of the vertices matching). Thus from a tiling of type $A$ if we delete all the short edges that join two vertices of different colors and have an $L_{A}$ and $S_{A}$ tile on either side we obtain a tiling of type $B$, that is, a tiling of the plane whose triangles are isometric to either $L_{B}$ or $S_{B}$ in Fig. 1. Again common vertices have same color (white or black) and common directed edges point in the same direction.

In the tiling of type $B$ so obtained, one never finds two $S_{B}$-triangles with a common directed long edge. In other words, on the other side of a directed long edge of an $S_{B}$-triangle of type $B$ tiling one always finds an $L_{B}$-triangle (with a matching directed edge). Deleting all such edges we obtain a new tiling $T_{1}$ of type $\tau A$, that is a tiling of the plane by triangles isometric to either $L_{\tau A}$ or $S_{\tau A}$ of Fig. 1 . The directed long edge in this inflated tile $L_{\tau A}$ points to the white vertex of the $S_{B}$-triangle in it. It only remains to change the color of the vertices of $L_{\tau A}$ and $S_{\tau A}$ from white (resp. black) to black (resp. white) so that $L_{\tau A}$ (resp. $S_{\tau A}$ ) becomes an inflation of $L_{A}$ (resp. $S_{A}$ ). The sides of $L_{\tau A}$ and $S_{\tau A}$ are proportional to the corresponding sides of $L_{A}$ and $S_{A}$ in the ratio $\tau$ to 1 , $\tau=(1+\sqrt{5}) / 2$. Iterating in this manner we get a sequence of tilings $T_{n}(n=0,1, \ldots)$ each of them having two prototiles $L_{n}, S_{n}$. The sides of $L_{n}$ and $S_{n}$ are proportional to those of $L_{0}$ and $S_{0}$ in the ratio $\tau^{n}$ to 1 .

Remark 3. To illustrate the proposed colorings, we suppose that if $\alpha$ and $\beta$ are two tiles of $T_{0}$, then, for sufficiently large $n, \alpha$ and $\beta$ are contained in the same tile of $T_{n}$. For a generic tiling this condition is satisfied. As pointed out in 10.5 .9 on p. 568 of [GS] there are exceptional tilings where the above condition is not satisfied. For such exceptional tilings the colorings of the kind proposed in this article can be obtained from another tiling where the condition is satisfied, by using the local isomorphism property [GS, 10.5.4, p. 562].

\subsection{Relative H-Orientation and Global H-Orientation of Tiles}

Let $H$ be a finite group and let $p$ be a positive integer. Let $\delta_{p, L}$ (resp. $\delta_{p, S}$ ) be a fixed long (resp. short) tile of the $T_{p}$-tiling. Let $\mathcal{U}_{\delta_{p, L}}$ (resp. $\mathcal{U}_{\delta_{p, S}}$ ) be the set of $T_{0}$-tiles of $\delta_{p, L}$ (resp. $\delta_{p, S}$ ).

Definition 4. A relative $H$-orientation of tiles is just a function

$$
\Psi_{H}: \mathcal{U}_{\delta_{p, L}} \cup \mathcal{U}_{\delta_{p, S}} \rightarrow H
$$

Given $\Psi_{H}$ as above, we define $\Psi_{H}(\delta) \in H$ for any tile $\delta$ of a $T_{m}$-tiling for any integer $m$ as follows. (We refer to $\Psi_{H}(\delta)$ as the relative $H$-orientation of $\delta$.) This tile $\delta$ is contained in a unique tile $\delta_{m+p}$ of the $T_{m+p}$-tiling. The relative position of $\delta$ in $\delta_{m+p}$ determines a unique element $\delta^{\prime}$ of $\mathcal{U}_{\delta_{p, L}}$ such that if $\delta^{\prime} \in \mathcal{U}_{\delta_{p, L}}\left(\operatorname{resp} . \mathcal{U}_{\delta_{p, S}}\right)$, then $\delta_{m+p}$ is large (resp. small) and the relative position of $\left(\delta, \delta_{m+p}\right)$ is similar to $\left(\delta^{\prime}, \delta_{p, L}\right)$ (resp. 
$\left(\delta^{\prime}, \delta_{p, S}\right)$ ) (if the first picture is drawn on a suitably smaller scale, it is exactly identical to the second picture). We set $\Psi_{H}(\delta)=\Psi_{H}\left(\delta^{\prime}\right)$.

Definition 5. Given a relative $H$-orientation as above, we say that a function $\Phi_{H}: \mathcal{U}_{0} \rightarrow$ $H\left(\mathcal{U}_{0}\right.$ is the set of $T_{0}$-tiles) is a global $H$-orientation of $T_{0}$-tiles, if the following property holds: for any two distinct $T_{0}$-tiles $\alpha$ and $\beta$, let $m$ be an integer such that $\alpha$ and $\beta$ belong to the same tile of $T_{m p}$ (here $p$ is the integer occurring in the definition of $\Psi_{H}$. We recall that our tilings are not exceptional (see Remark 3 )). Consider the sequence of tiles

$$
\alpha=\alpha_{0}, \alpha_{p}, \ldots, \alpha_{m p}=\beta_{m p}, \beta_{(m-1) p}, \ldots, \beta_{p}, \beta_{0}=\beta
$$

such that $\alpha_{i p}$ (resp. $\beta_{i p}$ ) is the unique tile of the $T_{i p}$-tiling which contains $\alpha_{(i-1) p}$ (resp. $\beta_{(i-1) p}$ ). The property we want (for a global $H$-orientation) is that

$\Psi_{H}\left(\alpha_{m p}\right) \cdots \Psi_{H}\left(\alpha_{p}\right) \cdot \Psi_{H}\left(\alpha_{0}\right) \cdot \Phi_{H}(\alpha)^{-1}=\Psi_{H}\left(\beta_{m p}\right) \cdots \Psi_{H}\left(\beta_{p}\right) \cdot \Psi_{H}\left(\beta_{0}\right) \cdot \Phi_{H}(\beta)^{-1}$.

As we show below (Proposition 7) the image of $\Phi_{H}$ is a subgroup $H^{\prime}$ of $H$. The same proposition also shows that the global $H$-orientation $\Phi_{H}$ is also associated to another relative $H$-orientation $\Psi_{H}^{(\ell)}$ whose image is $H^{\prime}$. In fact, $\Psi_{H}^{(\ell)}$ restricted to the subset of long (resp. short) $T_{0}$-tiles is onto $H^{\prime}$. We can assume without loss of generality that $H=H^{\prime}$.

Remark 6. Suppose that in some tiling $T$ (not necessarily the Robinson-Penrose tiling) there are only finitely many congruent classes of tiles and that the tiles congruent to any particular tile occur in only finitely many oriented positions in the usual sense of rotations by angles. (For example, this is the case for the Robinson-Penrose tilings, but not for the pinwheel tiling of Radin [R1].) Then, for an appropriately chosen finite subgroup $H$ of the orthogonal group, a global $H$-orientation $\Phi_{H}$ in the sense of Definition 5 can be given to coincide exactly with the usual sense of orientation. However, even for the pinwheel tiling, given any finite group $H$ and given any relative $H$-orientation $\Psi_{H}$, there always exist global $H$-orientations $\Phi_{H}$ such that the equation in Definition 5 is satisfied. For example, fix a $T_{0}$-tile $\alpha_{0}$, define $\Phi_{H}\left(\alpha_{0}\right)=e$ (the unit element of $H$ ), and for any $T_{0}$-tile $\beta$ define

$$
\Phi_{H}(\beta)=\Psi_{H}\left(\alpha_{0}\right)^{-1} \cdot \Psi_{h}\left(\alpha_{p}\right)^{-1} \cdots \Psi_{H}\left(\alpha_{m p}\right)^{-1} \cdot \Psi_{H}\left(\beta_{m p}\right) \cdots \Psi_{H}\left(\beta_{p}\right) \cdot \Psi_{H}\left(\beta_{0}\right),
$$

where we use the same notation as in Definition 5.

The purpose of the following proposition is to allow us to replace the relative $H$ orientation $\Psi_{H}$ by a new one with additional properties but whose associated global $H$-orientation remains the same. Let $\ell$ be a positive integer and let $\delta_{\ell p, L}$ (resp. $\delta_{\ell p, S}$ ) be a fixed long (resp. short) tile of the $T_{\ell p}$-tiling. Let $\mathcal{U}_{\delta_{\ell p, L}}$ (resp. $\mathcal{U}_{\delta_{\ell p, S}}$ ) be the set of $T_{0}$-tiles of $\delta_{\ell p, L}$ (resp. $\left.\delta_{\ell p, S}\right)$. Define

$$
\Psi_{H}^{(\ell)}: \mathcal{U}_{\delta_{\ell p, L}} \cup \mathcal{U}_{\delta_{\ell p, S}} \rightarrow H
$$

by

$$
\Psi_{H}^{(\ell)}(\beta)=\Psi_{H}\left(\beta_{(\ell-1) p}\right) \cdots \Psi_{H}\left(\beta_{p}\right) \cdot \Psi_{H}\left(\beta_{0}\right),
$$


where $\beta_{0}=\beta$ is any $T_{0}$-tile in $\mathcal{U}_{\delta_{\ell p, L}} \cup \mathcal{U}_{\delta_{\ell p, S}}$ and $\beta_{i p}$ is the unique tile of the $T_{i p}$-tiling which contains $\beta_{(i-1) p}$. Note (see Definition 4) that this allows us to define $\Psi_{H}^{(\ell)}(\delta) \in H$ for any tile $\delta$ of a $T_{m}$-tiling for any integer $m$ by looking at the relative position of $\delta$ in the unique $T_{m+\ell p}$-tile which contains $\delta$.

\section{Proposition 7.}

(i) Fix a $T_{0}$-tile $\alpha_{0}$ and define a global $H$-orientation $\Phi_{H}: \mathcal{U}_{0} \rightarrow H\left(\mathcal{U}_{0}\right.$ is the set of $T_{0}$-tiles) as in Remark 6. Define $\Phi_{H}^{(\ell)}$ in the same manner but replacing $p$ (resp. $\left.\Psi_{H}\right)$ by $\ell p\left(\right.$ resp. $\left.\Psi_{H}^{(\ell)}\right)$. Then $\Phi_{H}=\Phi_{H}^{(\ell)}$.

(ii) For any integer $n$, let $\alpha_{n p}$ be the unique $T_{n p}$-tile containing $\alpha_{0}$. Then

$$
\#\left(\Phi_{H}^{(\ell)}\left(\mathcal{U}_{\alpha_{\ell p}}\right)\right)=\#\left(\Psi_{H}^{(\ell)}\left(\mathcal{U}_{\alpha_{\ell p}}\right)\right) .
$$

(iii) Let $\ell$ be sufficiently large so that $\Phi_{H}\left(\mathcal{U}_{\alpha_{\ell p}}\right)=$ the image of $\Phi_{H}$ (note that such a choice of $\ell$ is possible since $\#(H)<+\infty$ and $\mathcal{U}_{0}$ is the union of $\mathcal{U}_{\alpha_{\ell p}}$ as $\ell$ varies). Then for any $T_{(\ell+1) p}$-tile $\delta_{(\ell+1) p}$ one has $\Phi_{H}^{(\ell+1)}\left(\mathcal{U}_{\delta_{(\ell+1) p}}\right)=$ the image of $\Phi_{H}$.

(iv) The image of $\Phi_{H}$ is a subgroup of $H$.

(v) Let $\ell$ be large so that $\Phi_{H}^{(\ell)}\left(\mathcal{U}_{\delta_{\ell p}}\right)=$ the image of $\Phi_{H}$ for any $T_{\ell p}$-tile $\delta_{\ell p}$. Let $k$ be the order of an element $g$ in the image of $\Psi_{H}^{(\ell)}$. Then the image of $\Psi_{H}^{(k \ell)}$ is a subgroup of $H$ and coincides with the image of $\Phi_{H}$.

(vi) Let $\ell^{\prime}=2 \ell$ where $\ell$ is as in $(\mathrm{v})$. For any $T_{\ell^{\prime} p}$-tile $\delta$ let $\mathcal{U}_{L, \delta}\left(\right.$ resp. $\left.\mathcal{U}_{S, \delta}\right)$ deote the set of long (resp. short) $T_{0}$-tiles in $\delta$. Then

$$
\Psi_{H}^{\left(\ell^{\prime}\right)}\left(\mathcal{U}_{\delta}\right)=\Psi_{H}^{\left(\ell^{\prime}\right)}\left(\mathcal{U}_{L, \delta}\right)=\Psi_{H}^{\left(\ell^{\prime}\right)}\left(\mathcal{U}_{S, \delta}\right)
$$

Proof. (i) With notation as in Definition 5, we have

$$
\Phi_{H}(\beta)=\Psi_{H}\left(\alpha_{0}\right)^{-1} \cdot \Psi_{H}\left(\alpha_{p}\right)^{-1} \cdots \Psi_{H}\left(\alpha_{m p}\right)^{-1} \cdot \Psi_{H}\left(\beta_{m p}\right) \cdots \Psi_{H}\left(\beta_{p}\right) \cdot \Psi_{H}\left(\beta_{0}\right) .
$$

Here $m$ can be replaced by any integer as this only introduces new terms which cancel off pairwise in the middle. Hence, the last quantity on the right side also equals

$$
\Psi_{H}^{(\ell)}\left(\alpha_{0}\right)^{-1} \cdot \Psi_{H}^{(\ell)}\left(\alpha_{\ell p}\right)^{-1} \cdots \Psi_{H}^{(\ell)}\left(\alpha_{m \ell p}\right)^{-1} \cdot \Psi_{H}^{(\ell)}\left(\beta_{m \ell p}\right) \cdots \Psi_{H}^{(\ell)}\left(\beta_{\ell p}\right) \cdot \Psi_{H}^{(\ell)}\left(\beta_{0}\right),
$$

which is nothing but $\Phi_{H}^{(\ell)}(\beta)$.

(ii) For a $T_{0}$-tile $\beta \in \mathcal{U}_{\alpha_{\ell p}}$ we have $\Phi_{H}^{(\ell)}(\beta)=g^{-1} \cdot \Psi_{H}^{(\ell)}(\beta)$ where $g=\Psi_{H}^{(\ell)}\left(\alpha_{0}\right)$.

(iii), (iv) Let $A^{(n)}=\Phi_{H}\left(\mathcal{U}_{\alpha_{n p}}\right)$. Then we have $A^{(1)} \subset A^{(2)} \subset \cdots A^{(n)} \subset \cdots \subset H$.

Choose $\ell$ so that $A^{(\ell)}=A^{(\ell)} \cup A^{(\ell+1)} \cup \cdots$. The tile $\alpha_{\ell p}$ may be a small or large tile, but any $T_{(\ell+1) p}$-tile $\delta$ contains a $T_{\ell p}$-tile congruent to $\alpha_{\ell p}$ (see Fig. 1). The proof of (iii) can be completed easily with this observation. Write $\ell^{\prime}=\ell+1$ and $B^{(n)}=\Psi_{H}^{(n)}\left(\mathcal{U}_{\alpha_{n p}}\right)$. From what we have demonstrated so far $B^{\left(\ell^{\prime}\right)}, B^{\left(2 \ell^{\prime}\right)}$, and $A^{\left(\ell^{\prime}\right)}$ all have the same cardinality. We claim that $B^{\left(\ell^{\prime}\right)} \cdot B^{\left(\ell^{\prime}\right)} \subset B^{\left(2 \ell^{\prime}\right)}$. Let $g, h \in B^{\left(\ell^{\prime}\right)}$. Let $\gamma$ be a $T_{0}$-tile in a $T_{\ell^{\prime}}$-tile $\delta$ whose relative $H$-orientation given by $\Psi_{H}^{\left(\ell^{\prime}\right)}$ is $g$. Let $\delta_{2 \ell^{\prime} p}$ be a $T_{2 \ell^{\prime} p}$-tile of the same tile type as $\delta$. Inside $\delta_{2 \ell^{\prime} p}$ let $\gamma_{\ell^{\prime} p}$ be a $T_{\ell^{\prime} p}$-tile whose relative position is identical to that of $\gamma$ inside 
$\delta$. Next, let $\beta$ be a $T_{0}$-tile inside $\gamma_{\ell^{\prime} p}$ such that $\Psi_{H}^{\left(\ell^{\prime}\right)}(\beta)=h$. Then $\Psi_{H}^{\left(2 \ell^{\prime}\right)}(\beta)=g h$, which proves our claim. Write $B$ for $B^{\left(\ell^{\prime}\right)}$. From the foregoing observations, we conclude that $\#(B)=\#(B \cdot B)$. Hence, if $b, b^{\prime} \in B$, then $b \cdot B=b^{\prime} \cdot B$. Thus, $b^{-1} b^{\prime} \cdot B=B$ for any $b$, $b^{\prime} \in B$. However, for any subset $B$ in any group $H$, the left stabilizer $\{g \in H \mid g \cdot B=B\}$ is a subgroup of $H$ and has cardinality at most equal to the cardinality of $B$. Thus, taking $B=B^{\left(\ell^{\prime}\right)}$ we conclude that $\{g \in H \mid g \cdot B=B\}=b^{-1} . B$ for any $b \in B$ and the latter is a subgroup of $H$. In particular, since the image of $\Phi_{H}$ is the same as $\Phi_{H}\left(\mathcal{U}_{\alpha_{\ell^{\prime} p}}\right)$ and since, $\forall \beta \in \mathcal{U}_{\alpha_{\ell^{\prime} p}}, \Phi_{H}(\beta)=\Psi_{H}^{\left(\ell^{\prime}\right)}\left(\alpha_{0}\right)^{-1} \cdot \Psi_{H}^{\left(\ell^{\prime}\right)}(\beta)$ we conclude that the image of $\Phi_{H}$ is a subgroup of $H$.

(v) By the same reasoning as was used above to show $B^{\left(\ell^{\prime}\right)} \cdot B^{\left(\ell^{\prime}\right)} \subset B^{\left(2 \ell^{\prime}\right)}$, we can also conclude that $B^{\left(\ell^{\prime}\right)} \cdot B^{\left(\ell^{\prime}\right)} \cdots B^{(\ell)}(k$-times $) \subset B^{(k \ell)}$. Since, by assumption, $g \in B^{(\ell)}$ has order $k$ it follows that $B^{(k \ell)}$ contains the identity. By the same reasoning as was used in the course of the proof of (iv) it can be shown that $b^{-1} \cdot \Psi_{H}^{(k \ell)}\left(\mathcal{U}_{\alpha_{k \ell p}}\right)$ is a subgroup of $H$ for any $b \in \Psi_{H}^{(k \ell)}\left(\mathcal{U}_{\alpha_{k \ell p}}\right)$. Hence, (v) follows.

(vi) Let $A_{L}^{(n)}$ (resp. $A_{S}^{(n)}$ ) be the image by $\Phi_{H}$ of the set of long (resp. short) tiles $\mathcal{U}_{L, \alpha_{n p}}\left(\operatorname{resp} . \mathcal{U}_{S, \alpha_{n p}}\right)$ in $\mathcal{U}_{\alpha_{n p}}$. Then we have

$$
A_{L}^{(1)} \subset A_{L}^{(2)} \subset \cdots A_{L}^{(n)} \subset \cdots \subset H
$$

and

$$
A_{S}^{(1)} \subset A_{S}^{(2)} \subset \cdots A_{S}^{(n)} \subset \cdots \subset H
$$

Choose $\ell$ large enough so that $A_{L}^{(\ell)}$ and $A_{S}^{(\ell)}$ are both maximal subsets in $H$. Then, similar to (iii), we find that for any $T_{(\ell+1) p}$-tile $\delta_{(\ell+1) p}$ one has $\Phi_{H}^{(\ell+1)}\left(\mathcal{U}_{\delta_{(\ell+1) p}}\right)=$ the image of $\Phi_{H}$ and furthermore $\Phi_{H}^{(\ell+1)}\left(\mathcal{U}_{L, \delta_{(\ell+1) p}}\right)=A_{L}^{(\ell)}$ and also $\Phi_{H}^{(\ell+1)}\left(\mathcal{U}_{S, \delta_{(\ell+1) p}}\right)=A_{S}^{(\ell)}$. Now write $B^{(n)}=\Psi_{H}^{(n)}\left(\mathcal{U}_{\alpha_{n p}}\right), B_{L}^{(n)}=\Psi_{H}^{(n)}\left(\mathcal{U}_{L, \alpha_{n p}}\right)$, and $B_{S}^{(n)}=\Psi_{H}^{(n)}\left(\mathcal{U}_{S, \alpha_{n p}}\right)$. Write $\ell^{\prime}=\ell+1$. By the same argument used in the course of the proof of (iv), we conclude that $B^{\left(\ell^{\prime}\right)} \cdot B_{L}^{\left(\ell^{\prime}\right)} \subset B_{L}^{\left(2 \ell^{\prime}\right)}$, but $B_{L}^{\left(\ell^{\prime}\right)} \subset B^{\left(\ell^{\prime}\right)}$. The maximality of \# $\left(B_{L}^{\left(\ell^{\prime}\right)}\right)$ and \# $\left(B^{\left(\ell^{\prime}\right)}\right)$ now implies that $\#\left(B_{L}^{\left(\ell^{\prime}\right)}\right)=\#\left(B^{\left(\ell^{\prime}\right)}\right)=\#\left(B_{L}^{\left(2 \ell^{\prime}\right)}\right)$. This completes the proof of (vi) for long tiles. Short tiles can be handled similarly.

Definition 8 (Color Isolation). When a global $H$-orientation $\Phi_{H}$ by elements of a group $H$ is given we could also consider colorings where tiles are permitted to receive only those colors allowed by their global $H$-orientation. More precisely let $G$ be a finite group acting on a finite set $M: \sigma: G \times M \rightarrow M$. For each $h \in H$ let $M_{h}$ be a nonempty subset of $M$ stable under $G$. The coloring of $T_{0}$ is said to be color isolated if any tile in global $H$-orientation $h$ has a color from $M_{h}$. When color isolation is not prescribed we refer to the coloring as "mixed coloring."

\subsection{Mixed Coloring in the Case $G=\operatorname{Aut}(M)$}

The mixed coloring in the particular case when $G$ is the full permutation group $\operatorname{Aut}(M)$ of the set $M$ of colors, is our goal in this subsection. Thus we have a finite set $M=\{1, \ldots, k\}$ and $G=\mathfrak{S}_{k}$, the group of all permutations of $\{1, \ldots, k\} ; T_{0}$ is a given tiling of the plane 
as in Section 1. Let $\mathcal{U}_{0}$ be the set of all tiles of $T_{0}$. We want to describe a function (assignment of a color to a tile)

$$
\varphi: \mathcal{U}_{0} \rightarrow\{1, \ldots, k\}
$$

We do this by first defining $\tilde{\varphi}: \mathcal{U}_{0} \rightarrow \mathfrak{S}_{k}$ and then setting, for $\delta \in \mathcal{U}_{0}, \varphi(\delta)=\tilde{\varphi}(\delta) \cdot 1$ (the permutation $\tilde{\varphi}(\delta)$ applied to 1 ).

We now begin the task of defining $\tilde{\varphi}: \mathcal{U}_{0} \rightarrow \mathfrak{S}_{k}$. We choose an integer $p$. Let $\delta_{p, L}$ (resp. $\delta_{p, S}$ ) be any large (resp. small) $T_{p}$-tile. Now fix $\delta_{p, L}$ and $\delta_{p, S}$. Let

$$
\mathcal{U}_{\delta_{p, L}}=\text { set of } T_{0} \text {-tiles contained in } \delta_{p, L}
$$

and

$$
\mathcal{U}_{\delta_{p, S}}=\text { set of } T_{0} \text {-tiles contained in } \delta_{p, S}
$$

Remark 9. Evidently, if $p$ is large enough we can choose a function

$$
\Psi: \mathcal{U}_{\delta_{p, L}} \cup \mathcal{U}_{\delta_{p, S}} \rightarrow \mathfrak{S}_{k}
$$

such that $\Psi\left(\mathcal{U}_{L, \delta}\right)=\mathfrak{S}_{k}=\Psi\left(\mathcal{U}_{S, \delta}\right)$ where $\delta=\delta_{p, L}$ or $\delta_{p, S}$ and $\mathcal{U}_{L, \delta}\left(\right.$ resp. $\left.\mathcal{U}_{S, \delta}\right)$ stands for the set of long (resp. short) $T_{0}$-tiles in $\delta$.

For any integer $m$ and any tile $\delta_{m}$ of the $T_{m}$-tiling we can define $\Psi\left(\delta_{m}\right) \in \mathfrak{S}_{k}$ following the method explained in Definition 4.

Fix a tile $\alpha$ of the $T_{0}$-tiling. Choose an arbitrary element $h_{0}$ of $H$. Now let $\beta$ be any tile of $T_{0}$. Let $m$ be an integer such that $\alpha$ and $\beta$ belong to the same tile of $T_{m p}$. Let

$$
\alpha=\alpha_{0}, \alpha_{p}, \ldots, \alpha_{m p}=\beta_{m p}, \ldots, \beta_{p}, \beta_{0}=\beta
$$

be chosen such that $\alpha_{i p}$ (resp. $\beta_{i p}$ ) is the unique tile of the $T_{i p}$-tiling which contains $\alpha_{(i-1) p}\left(\right.$ resp. $\beta_{(i-1) p}$. We define

$$
\tilde{\varphi}(\alpha)=h_{0}
$$

and

$$
\tilde{\varphi}(\beta)=h_{0} \cdot \Psi\left(\alpha_{0}\right)^{-1} \cdot \Psi\left(\alpha_{p}\right)^{-1} \cdots \Psi\left(\alpha_{m p}\right)^{-1} \cdot \Psi\left(\beta_{m p}\right) \cdots \Psi\left(\beta_{p}\right) \cdot \Psi\left(\beta_{0}\right) .
$$

With these preparations we are now ready to indicate the proof of Theorem $2((G, \sigma, M)$ symmetry for $G=\operatorname{Aut}(M))$ stated in the Introduction.

We will now prove that the color assignment $\varphi(\beta)=\tilde{\varphi}(\beta) \cdot 1\left(\beta \in \mathcal{U}_{0}\right)$ has the local $(G, M)$-symmetry property (see Definition 1 in the Introduction) for $G=\mathfrak{S}_{k}$, $M=\{1, \ldots, k\}$.

Let $\Sigma$ be a finite patch of $T_{0}$-tiles. Let $\sigma \in \mathfrak{S}_{k}$ be given. We can assume that $\Sigma$ is contained in a tile $\delta_{l p}$ of the $T_{l p}$-tiling for $l$ sufficiently large. (This is possible since $T_{0}$ is not an exceptional tiling (see Remark 3).) Let $\delta_{(l+1) p}^{\prime}$ be any $T_{(l+1) p}$-tile. We are going to find a $T_{l p}$-tile $\delta_{l p}^{\prime}$ contained in $\delta_{(l+1) p}^{\prime}$ having the same tile type (i.e., large or small) as $\delta_{l p}$ such that the color of a $T_{0}$-tile of $\delta_{l p}^{\prime}$ is related to the color of the corresponding $T_{0}$-tile of $\delta_{l p}$ via the permutation $\sigma$. It is clear that such a tile $\delta_{l p}^{\prime}$ exists because if $\mathcal{U}_{l}^{\prime}$ is 
the set of all $T_{l p}$-tiles of the same tile type as $\delta_{l p}$ contained in $\delta_{(l+1) p}^{\prime}$, by the choice of $\Psi, \Psi\left(\mathcal{U}_{l}^{\prime}\right)=\mathfrak{S}_{k}$.

Therefore, the color assignment given by $\varphi$ has the local $\left(\mathfrak{S}_{k},\{1, \ldots, k\}\right)$-symmetry property.

As we pointed out in the Introduction, in order to produce a (mixed) coloring with $(G, \sigma, M)$-symmetry it suffices trivially to handle the case when $G$ is the full automorphism group of $M$. Thus we have found a coloring of the $T_{0}$-tiles which has local $(G, M)$-symmetry.

\subsection{Colorings with Prescribed Isolation and Frequencies}

Recall Definition 8 of "color isolation." We now prove the existence of a coloring of $T_{0}$ with $(G, \sigma, M)$-symmetry with the additional requirement of color isolation.

More precisely, let $\Phi_{H}: \mathcal{U}_{0} \rightarrow H$ be a global $H$-orientation of $T_{0}$-tiles $\left(\mathcal{U}_{0}\right.$ is the set of $T_{0}$-tiles). Let $M_{h}(h \in H)$ be $G$-stable subsets of $M$ consisting of the allowed colors for tiles in global $H$-orientation $h$. Let $S$ be the set $\prod_{h \in H} M_{h}$. The group $G$ acts on $S$ coordinatewise. Let $\#(S)=k$. Fix a bijection of $S$ with $\{1, \ldots, k\}$ and proceed as in the previous section to get a coloring $\varphi$ of $\mathcal{U}_{0}$ by elements of $S$ with $\operatorname{Aut}(S)$-symmetry. Let $\delta \in \mathcal{U}_{0}$. Let $h$ be the global $H$-orientation of $\delta$. We define

$$
\varphi^{\prime}: \mathcal{U}_{0} \rightarrow M
$$

by $\varphi^{\prime}(\delta)=$ the $h$-coordinate of $\varphi(\delta)$. This is an isolated coloring of the tiles of $T_{0}$ by the colors in $M$ and has local $(G, M)$-symmetry.

Finally, as we stated in the Introduction, in addition to color isolation one may also wish to see colors in certain frequencies by prescribing the desired ratios. More precisely, let $M_{h}(h \in H)$ be $G$-stable subsets of $M$ as above consisting of the allowed colors for tiles in global $H$-orientation $h$. Let $C_{h, 1}, \ldots, C_{h, N}$ be the distinct $G$-orbits in $M_{h}$. (Of course, $N$ depends on $h$.) Suppose that one wants to see the colors of $C_{h, 1}, \ldots, C_{h, N}$ in the frequency ratios $a_{h, 1}, \ldots, a_{h, N}$ (positive integers) among the tiles in global $\mathrm{H}$ orientation $h$. (What we mean by this statement, if not already obvious, will become clear in the statement of Theorem 10 below). Let $S_{h}^{\prime}$ be a set which is a disjoint union of $a_{h, 1}$ copies of $C_{h, 1}, a_{h, 2}$ copies of $C_{h, 2}, \ldots, a_{h, N}$ copies of $C_{h, N}$. Let $S^{\prime}$ be the set $\prod_{h \in H} S_{h}^{\prime}$. The group $G$ acts on each $S_{h}^{\prime}$ and hence on $S^{\prime}$. Let $\#\left(S^{\prime}\right)=k$. Fix a bijection of $S^{\prime}$ with $\{1, \ldots, k\}$ and identify $\left(\operatorname{Aut}\left(S^{\prime}\right),\left(S^{\prime}\right)\right)$ with $\left(\mathfrak{S}_{k},\{1, \ldots, k\}\right)$. Proceed as before to get first a coloring $\varphi$ of $\mathcal{U}_{0}$ by elements of $S^{\prime}$ with $\operatorname{Aut}\left(S^{\prime}\right)$-symmetry. Let $\delta \in \mathcal{U}_{0}$. Let $h$ be the global $H$-orientation of $\delta$. We define

$$
\varphi^{\prime}: \mathcal{U}_{0} \rightarrow M
$$

by $\varphi^{\prime}(\delta)=$ the $h$-coordinate of $\varphi(\delta)$. (Strictly speaking, the $h$-coordinate of $\varphi(\delta)$ is an element of $S_{h}^{\prime}$, but the latter is mapped in an obvious way to $M$ as $S_{h}^{\prime}$ is a union of several copies of subsets of $M$.)

The integer $p$ in the statement of the theorem below is a sufficiently large multiple of the integer (with the same symbol) which appears in Definition 4 and also the one chosen as in Remark 9 after having identified $\left(\operatorname{Aut}\left(S^{\prime}\right),\left(S^{\prime}\right)\right)$ with $\left(\mathfrak{S}_{k},\{1, \ldots, k\}\right)$ as above. 
Theorem 10. The above coloring of $T_{0}$ which is associated to a frequency data $a_{*}=$ $\left\{\left(a_{h, 1}, \ldots, a_{h, i}, \ldots, a_{h, N}\right) \mid h \in H\right\}$ has the following properties:

(i) It has local $(G, \sigma, M)$-symmetry.

(ii) Let $\alpha$ be a fixed $T_{0}$-tile. Let $\delta_{m p}$ be the unique $T_{m p}$-tile which contains $\alpha$. Let $h \in H$ and let $C_{h, i}, C_{h, j}$ be two G-orbits in $M_{h}$. Let $\mu \in C_{h, i}$. Let $\mu^{\prime} \in C_{h, j}$. Let $A_{h, \mu}\left(\delta_{m p}\right)$ (resp. $\left.A_{h, \mu^{\prime}}\left(\delta_{m p}\right)\right)$ be the number of $T_{0}$-tiles of $\delta_{m p}$ in global $\mathrm{H}$ orientation $h$ and having color $\mu$ (resp. $\left.\mu^{\prime}\right)$. Then $A_{h, \mu}\left(\delta_{m p}\right) / A_{h, \mu^{\prime}}\left(\delta_{m p}\right)$ tends to $a_{h, i} / a_{h, j}$ as $m \rightarrow+\infty$.

(iii) Let $h, h^{\prime} \in H$. Let $A_{h}\left(\delta_{m p}\right)$ (resp. $A_{h^{\prime}}\left(\delta_{m p}\right)$ ) be the number of $T_{0}$-tiles of $\delta_{m p}$ in global $H$-orientation $h\left(\right.$ resp. $\left.h^{\prime}\right)$. Then $A_{h}\left(\delta_{m p}\right) / A_{h^{\prime}}\left(\delta_{m p}\right)$ tends to 1 as $m \rightarrow$ $+\infty$.

We have already noted that the coloring has local $(G, \sigma, M)$-symmetry. The remaining part of the article is devoted to the proof of the other two assertions in the theorem.

\section{Uniform Distribution of Colors and $\boldsymbol{H}$-Orientations}

We begin with a lemma on the group algebra of a finite group. Let $\mathfrak{S}$ be a finite group and let $\mathbb{R}[\mathfrak{S}]$ be the group algebra of $\mathfrak{S}$ over $\mathbb{R}$. It has a natural topology as a Euclidean space. Define a subset $K$ of $\mathbb{R}[\mathfrak{S}]$ by

$$
K=\left\{\sum_{s \in \mathfrak{S}} a_{s} s: a_{s} \geq 0, \forall s \text { and } \sum_{s \in \mathfrak{S}} a_{s}=1\right\} .
$$

The subset $K$ is compact. Note that $K \cdot K \subset K$.

In the statement of the two lemmas which follow, part (i) can be deduced from the Perron-Frobenius theorem (see p. 53 of [Ga], for example). This was pointed out to us by the referee for which we are thankful. For the sake of completeness we have indicated a proof and this also serves another purpose: our proof of part (ii) of either lemma requires only a slight modification of the arguments in part (i).

\section{Lemma 11.}

(i) Let $b=\sum_{s \in \mathfrak{S}} b_{s} s$ be an element of the group algebra of $\mathfrak{S}$ over $\mathbb{R}$. Assume $b \in K$ and $b_{s}>0, \forall s$. If $b^{m}=\sum_{s \in \mathfrak{S}} \alpha_{m, s} \cdot s$, then $\alpha_{m, s}$ tends to $1 / \#(\mathfrak{S})$ as $m \rightarrow+\infty$.

(ii) Let $b_{n}=\sum_{s \in \mathfrak{S}} b_{s, n} s$ be a sequence of elements of $K$. Assume that $\lim _{n \rightarrow+\infty} b_{n}=$ $b$ exists and $b=\sum_{s \in \mathfrak{S}} b_{s} s$ where $b_{s}>0, \forall s$. Let $x \in K$. Define a sequence $\left\{x_{n}\right\}$ by

$$
x_{1}=b_{1} x, \quad x_{2}=b_{2} x_{1}, \quad \ldots, \quad x_{n}=b_{n} x_{n-1}, \ldots
$$

Then $\lim _{n \rightarrow+\infty} x_{n}=\sum_{s \in \mathfrak{S}}(1 / \#(\mathfrak{S})) s$.

Proof. (i) Let $K_{0}$ be the intersection of all the closures $\overline{\left\{b_{m}: m \geq n\right\}}$. Choose $\alpha=$ $\sum a_{g} g \in K_{0}$ such that, for every $\gamma=\sum c_{g} g \in K_{0}, \sup _{g}\left(c_{g}-1 / \#(\mathfrak{S})\right) \geq \sup _{g}\left(a_{g}-\right.$ $1 / \#(\mathfrak{S}))$ (since $K_{0}$ is compact such an $\alpha$ exists). 
Now let $\alpha^{\prime}=b \alpha$. Write $\alpha^{\prime}=\sum a_{g}^{\prime} g$. Then $a_{g}^{\prime}=\sum_{s \in \mathfrak{S}} b_{s^{-1}} a_{s g}$ and

$$
\begin{aligned}
a_{g}^{\prime}-\frac{1}{\#(\mathfrak{S})} & =\sum_{s} b_{s^{-1}} a_{s g}-\sum_{s} b_{s^{-1}}\left(\frac{1}{\#(\mathfrak{S})}\right) \\
& =\sum_{s} b_{s^{-1}}\left(a_{s g}-\frac{1}{\#(\mathfrak{S})}\right) \\
& \leq \sum_{s} b_{s^{-1}} \sup _{k}\left(a_{k}-\frac{1}{\#(\mathfrak{S})}\right) \\
& =\sup _{k}\left(a_{k}-\frac{1}{\#(\mathfrak{S})}\right)
\end{aligned}
$$

with strict inequality if $\left(a_{k}-1 / \#(\mathfrak{S})\right)$ are not all equal. By the choice of $\alpha$ strict inequality is not possible. Hence $a_{k}-1 / \#(\mathfrak{S})$ is independent of $k \in \mathfrak{S}$. However, $1 / \#(\mathfrak{S})$ is the average of $\left\{a_{k}: k \in \mathfrak{S}\right\}$ as $\sum_{k} a_{k}=1$. Hence $a_{k}=1 / \#(\mathfrak{S})$; which implies $a_{g}^{\prime}=1 / \#(\mathfrak{S})$, i.e., $b \alpha=\alpha$.

Since $\alpha \in K_{0}$ we can choose a subsequence $\left(b^{n_{i}}\right)_{i \in \mathbb{N}^{*}}$ such that

$$
\lim _{i \rightarrow+\infty} b^{n_{i}}=\alpha=\frac{1}{\#(\mathfrak{S})} \sum_{s} s .
$$

Thus if $b^{n_{i}}=\sum b_{n_{i, s}} s$, then $b_{n_{i, s}}>0, \forall i, s$, and $b_{n_{i, s}} \rightarrow 1 / \#(\mathfrak{S})$.

For any $m$ let $b^{m}=\sum b_{m, s} S$. Then $\sum b_{m+1, s} \cdot s=\left(\sum b_{s} \cdot s\right)\left(\sum b_{m, s} \cdot s\right)$. So

$$
b_{m+1, s}-b_{m+1, s^{\prime}}=\sum_{g \in \mathfrak{S}} b_{g^{-1}}\left(b_{m, g s}-b_{m, g s^{\prime}}\right) \text {. }
$$

Hence

$$
\begin{aligned}
\sup _{s, s^{\prime}}\left|b_{m+1, s}-b_{m+1, s^{\prime}}\right| & \leq\left(\sum_{g \in \mathfrak{S}} b_{g^{-1}}\right) \sup _{s, s^{\prime}}\left|b_{m, s}-b_{m, s^{\prime}}\right| \\
& =\sup _{s, s^{\prime}}\left|b_{m, s}-b_{m, s^{\prime}}\right| .
\end{aligned}
$$

From this inequality together with the fact that $\lim _{i \rightarrow+\infty} b^{n_{i}}=(1 / \#(\mathfrak{S})) \sum_{s} s$ we obtain that $\lim _{n \rightarrow+\infty} b^{n}=(1 / \#(\mathfrak{S})) \sum_{s} s$.

(ii) Let $K_{0}$ be the intersection over $n$ of all the closures of $\left\{x_{m}: m \geq n\right\}$. Choose $\alpha=\sum a_{g} g \in K_{0}$ such that, for every $\gamma=\sum c_{g} g \in K_{0}$,

$$
\sup _{g}\left(c_{g}-\frac{1}{\#(\mathfrak{S})}\right) \geq \sup _{g}\left(a_{g}-\frac{1}{\#(\mathfrak{S})}\right) .
$$

We claim that $a_{g}=1 / \#(\mathfrak{S})$ for all $g$. Suppose that all the $a_{g}$ are not equal. Let $\left.\tau=\left(\sup \left(a_{g}\right)\right)-\inf \left(a_{g}\right)\right)$. So $\tau>0$. Let $\varepsilon$ be a small positive real number. We can choose an integer $N>>0$ such that, for all $n>N$ and all $s \in \mathfrak{S}$, we have

$$
b_{s, n} \geq \inf _{s}\left(b_{s}\right)-\varepsilon
$$


$\operatorname{~inf}_{s}\left(b_{s}\right)$ is positive by the assumption made in the statement of part (ii) of the lemma). Write $x_{n}=\sum_{s} a_{s, n} s$. Since $x_{n+1}=b_{n+1} \cdot x_{n}$ we have

$$
\begin{aligned}
a_{g, n+1}-\frac{1}{\#(\mathfrak{S})} & =\sum_{s} b_{s^{-1}, n+1}\left(a_{s g, n}-\frac{1}{\#(\mathfrak{S})}\right) \\
& \leq \sup \left(a_{s g, n}-\frac{1}{\#(\mathfrak{S})}\right)-b_{w^{-1}, n+1}\left(\sup _{s}\left(a_{s g, n}\right)-\left(a_{w g, n}\right)\right) \quad(\forall w) \\
& \leq \sup \left(a_{s g, n}-\frac{1}{\#(\mathfrak{S})}\right)-\left(\inf _{s}\left(b_{s}\right)-\varepsilon\right)(\tau-\varepsilon) \quad(\text { if } n>>0) \\
& \leq \sup \left(a_{s}-\frac{1}{\#(\mathfrak{S})}\right)+\varepsilon-\left(\inf _{s}\left(b_{s}\right)-\varepsilon\right)(\tau-\varepsilon) \quad(\text { if } n>>0) .
\end{aligned}
$$

Obviously it is possible to choose $\varepsilon$ small enough such that

$$
\left(\inf \left(b_{s}\right)-\varepsilon\right)(\tau-\varepsilon)-\varepsilon \geq \frac{1}{2}\left(\inf \left\{\tau, \inf _{s}\left(b_{s}\right)\right\}\right)^{2} .
$$

With such a choice of $\varepsilon$, we obtain

$$
a_{g, n+1}-\frac{1}{\#(\mathfrak{S})} \leq \sup a_{s}-\frac{1}{\#(\mathfrak{S})}-\frac{1}{2}\left(\inf \left\{\tau, \inf \left(b_{s}\right)\right\}\right)^{2} .
$$

Thus if a subsequence $\left\{x_{n_{1}}, x_{n_{2}}, \ldots, x_{n_{j}}, \ldots\right\}$ has limit $\alpha=\sum a_{s} s \in K_{0}$, then the subsequence $\left\{x_{1+n_{1}}, x_{1+n_{2}}, \ldots, x_{1+n_{j}}, \ldots\right\}$ has a limit $\gamma=\sum c_{s} s$, where

$$
\sup _{s}\left(c_{s}-\frac{1}{\#(\mathfrak{S})}\right)<\sup _{s}\left(a_{s}-\frac{1}{\#(\mathfrak{S})}\right) \text {. }
$$

However, this contradicts $(*)$. This completes the proof that $a_{s}=1 / \#(\mathfrak{S})$ for all $s$.

Now, the proof that $\lim _{n \rightarrow+\infty} x_{n}=\sum_{s}(1 / \#(\mathfrak{S})) s$ can be completed as in part (i).

The aim of the next lemma is to arrive at the same conclusion as in the previous lemma about the limit of a more complicated sequence.

\section{Lemma 12.}

(i) Let $E=\left(x_{i, j}\right)_{1 \leq i, j \leq k}$ be a square matrix whose entries are elements of the group algebra of $\mathfrak{S}$ over $\mathbb{R}\left(\mathfrak{S}\right.$ is a finite group). Let $\left(x_{i}\right)_{1 \leq i \leq k}$ be a column vector defined by $x_{i}=\sum_{j=1}^{k} x_{i, j}$. Assume that $x_{i} \in K, \forall i$. Let $b^{(0)}=\left(b_{i}^{(0)}\right)_{1 \leq i \leq k}$ be a column vector of elements of $K$. Define $b^{(m)}=E^{m} \cdot b^{(0)}$. Then the entries of $b^{(m)}$ belong to $K$. If, for each $s \in \mathfrak{S}$, the coefficient of $s$ in each $x_{i, j}$ is positive, then

$$
\lim _{m \rightarrow+\infty} b^{(m)}=\left(\bar{b}_{i}\right)_{1 \leq i \leq k}
$$

where each $\bar{b}_{i}$ equals the element $\left(\sum_{s \in \mathfrak{S}} s\right) / \#(\mathfrak{S})$. 
(ii) Let $E(n)=\left(x_{i, j}(n)\right)$ be a sequence of $k \times k$ matrices whose entries are elements of the group algebra of $\mathfrak{S}$ over $\mathbb{R}$. Assume that $\lim _{n \rightarrow+\infty} E(n)=E=\left(x_{i, j}\right)$ exists. For each $n$ define the column vector $\left(x_{i}(n)\right)_{1 \leq i \leq k}$ by $x_{i}(n)=\sum_{j=1}^{k} x_{i, j}(n)$. Assume that $x_{i}(n) \in K \forall i, n$. Let $b^{(0)}=\left(b_{i}^{(0)}\right)_{1 \leq i \leq k}$ be a column vector of elements of $K$. Define, inductively, $b^{(m)}=E(m) b^{(m-1)}$. Then the elements of $b^{(m)}$ belong to $K$. If, for each $s \in \mathfrak{S}$, the coefficient of $s$ in each $x_{i, j}$ is positive, then

$$
\lim _{n \rightarrow+\infty} b^{(n)}=\left(\bar{b}_{i}\right)_{1 \leq i \leq k}
$$

where each $\bar{b}_{i}$ equals $\left(\sum_{s \in \mathfrak{S}} s\right) / \#(\mathfrak{S})$.

Proof. (i) Let $K_{0}$ be the intersection of all the closures $\overline{\left\{b^{(m)}: m \geq n\right\}}$. Then $K_{0}$ is a compact set of $K \times \cdots \times K(k$ copies of $K)$. We will show that $K_{0}$ consists of the single point mentioned in the statement of the lemma.

Choose $\mu=\left(\mu_{i}\right)_{1 \leq i \leq k} \in K_{0}$ with the following property: For every $v=\left(v_{i}\right)_{1 \leq i \leq k} \in$ $K_{0}$, writing $\mu_{i}=\sum a_{i, g} g$ and $v_{i}=\sum c_{i, g} g$,

$$
\sup _{i, g}\left(c_{i, g}-\frac{1}{\# \mathfrak{S}}\right) \geq \sup _{i, g}\left(a_{i, g}-\frac{1}{\# \mathfrak{S}}\right) .
$$

Now let $\mu^{\prime}=E \cdot \mu$. Let $\mu^{\prime}=\left(\mu_{i}^{\prime}\right)$ and write $\mu_{i}^{\prime}=\sum a_{i, g}^{\prime} g$. Then $\mu_{i}^{\prime}=\sum_{j} x_{i, j} \mu_{j}$ and writing $x_{i, j}=\sum_{s} x_{i, j, s} s$, one has

$$
a_{i, g}^{\prime}=\sum_{j, s} x_{i, j, s^{-1}} a_{j, s g}
$$

Hence

$$
\begin{aligned}
a_{i, g}^{\prime}-\frac{1}{\# \mathfrak{S}} & =\sum_{j, s} x_{i, j, s^{-1}} a_{j, s g}-\sum_{j, s} x_{i, j, s^{-1}} \frac{1}{\# \mathfrak{S}} \\
& =\sum_{j s} x_{i, j, s^{-1}}\left(a_{j, s g}-\frac{1}{\# \mathfrak{S}}\right) \\
& \leq \sum_{j, s} x_{i, j, s^{-1}} \sup _{j, g^{\prime}}\left(a_{j, g^{\prime}}-\frac{1}{\# \mathfrak{S}}\right) .
\end{aligned}
$$

Since $x_{i, j, s}>0, \forall(i, j, s)$, and since $\sum_{j, s} x_{i, j, s}=1$ the inequality will be strict if $\left(a_{j, g^{\prime}}-1 / \# \mathfrak{S}\right)$ are not equal. By the choice of $\mu$, strict inequality is not possible. This implies that $\mu=\bar{b}$ as asserted and that $E \mu=E \bar{b}=\bar{b}$.

We can choose a subsequence $\left\{b^{\left(n_{i}\right)}\right\}_{i=1,2, \ldots}$ such that $\lim _{n \rightarrow+\infty} b^{\left(n_{i}\right)}=\bar{b}$. For any $m$, let $b^{(m)}=\left(b_{j}^{(m)}\right)_{1 \leq i \leq k}$ where $b_{j}^{(m)}=\sum_{g} b_{j, g}^{(m)} g$ (the sum is over $g \in \mathfrak{S}$ ). Then

$$
b_{j}^{(m+1)}=\sum_{l} x_{j, l}, b_{l}^{(m)}
$$


and

$$
b_{j, s}^{(m+1)}=\sum_{l, g} x_{j, l, g^{-1}} b_{l g, s}^{(m)}
$$

Hence

$$
b_{j, s}^{(m+1)}-b_{j, s^{\prime}}^{(m+1)}=\sum_{l, g} x_{j, l, g^{-1}}\left(b_{l, g s}^{(m)}-b_{l, g s^{\prime}}^{(m)}\right) .
$$

Hence

$$
\begin{aligned}
\sup _{s, s^{\prime} \in \mathfrak{S}}\left|b_{j, s}^{(m+1)}-b_{j, s^{\prime}}^{(m+1)}\right| & \leq\left(\sum_{l, g} x_{j, l, g^{-1}}\right) \cdot \sup _{s, s^{\prime} \in \mathfrak{S}, 1 \leq l \leq k}\left|b_{l, s}^{(m)}-b_{l, s^{\prime}}^{(m)}\right| \\
& =\sup _{s, s^{\prime} \in \mathfrak{S}, 1 \leq l \leq k}\left|b_{l, s}^{(m)}-b_{l, s^{\prime}}^{(m)}\right| .
\end{aligned}
$$

From this inequality together with the fact that $\lim _{i \rightarrow+\infty} b^{\left(n_{i}\right)}=\left(\bar{b}_{j}\right)_{1 \leq j \leq k}$ where $\bar{b}_{j}$ is equal to $\left(\sum_{g \in \mathfrak{S}} g\right) / \# \mathfrak{S}$ it follows that $\lim _{n \rightarrow+\infty} b^{(n)}=\left(\bar{b}_{j}\right)$.

(ii) The proof of this part only requires the above argument to be slightly modified in the same way as the second part of Lemma 11.

\subsection{An Application of Lemma 12}

We now use the above lemmas to describe some results about uniform distributions. (See [R2] for rules which force uniform orientational distributions of the tiles in a different sense and for a study of ergodic properties of tilings.)

Let $\mathfrak{S}$ be a finite group and let $\Psi: \mathcal{U}_{\delta_{p, L}} \cup \mathcal{U}_{\delta_{p, S}} \rightarrow \mathfrak{S}$ be a function as in Definition 4 . For any integer $m \geq 0$ and for any tile $\delta_{m p}$ of the $T_{m p}$-tiling define $\Psi\left(\delta_{m p}\right) \in \mathfrak{S}$ as in Definition 4 , by looking at the relative position of $\left(\delta_{m p}, \delta_{(m+1) p}\right)$ where $\delta_{(m+1) p}$ is the unique tile of $T_{(m+1) p}$ which contains $\delta_{m p}$.

We now define two elements $b\left(\delta_{m p}\right), B\left(\delta_{m p}\right) \in \mathbb{R}[\mathfrak{S}]$ of the group algebra of $\mathfrak{S}$ over $\mathbb{R}$ as follows: $b()$ is obtained by normalizing $B()$ :- if $B()=\sum A_{s} s, b=\sum\left(A_{s} / A\right) s$ where $A=\sum A_{s}$. Clearly $b() \in K$, where $K$ is the compact space defined just before Lemma 11. $B($ ) is defined inductively as follows:

(i) $m=0$. For a tile $\delta$ of the $T_{0}$-tiling $B(\delta)=e$, the unit element of $\mathbb{R}[\mathfrak{S}]$.

(ii) Suppose $B\left(\mathrm{)}\right.$ has been defined for all the tiles of the $T_{i p}$-tiling for $i<m$. We define

$$
B\left(\delta_{m p}\right)=\sum \Psi\left(\delta_{(m-1) p}\right) \cdot B\left(\delta_{(m-1) p}\right)
$$

where $\Psi(\delta)$ is as in Definition 4 and the sum is over all the tiles of the $T_{(m-1) p^{-}}$ tiling contained in $\delta_{m p}$. 
Remark 13. For a $T_{0}$-tile $\delta_{0}$ of $\delta_{m p}$, let $\delta_{0}, \delta_{1}, \ldots, \delta_{(m-1) p}$ be the sequence such that $\delta_{i p}$ is the unique $T_{i p}$-tile which contains $\delta_{(i-1) p}$. Then it is easy to see that

$$
B\left(\delta_{(m p)}=\sum_{\delta_{0}} \Psi\left(\delta_{(m-1) p}\right) \cdots \cdots \Psi\left(\delta_{p}\right) \cdot \Psi\left(\delta_{0}\right),\right.
$$

where the sum is taken over all the $T_{0}$-tiles $\delta_{0}$ contained in $\delta_{m p}$.

Clearly, $B\left(\delta_{m p}\right)$ depends only on $m$ and whether $\delta_{m p}$ is large or small. Normalizing this for each $m$ we have two elements $b_{m, L}$ and $b_{m, S}$ of $K$.

Lemma 14. We have

$$
\lim _{m \rightarrow+\infty} b_{m, L}=\lim _{m \rightarrow+\infty} b_{m, S}=\frac{1}{\#(\mathfrak{S})} \sum_{s \in \mathfrak{S}} s .
$$

Proof. Let $B_{1, L}=B\left(\delta_{p, L}\right)$ where $\delta_{p, L}$ is a long tile of $T_{p}$. Then $B_{1, L}=\sum_{\delta_{0}} \Psi\left(\delta_{0}\right)$ where the sum is over all the $T_{0}$-tiles $\delta_{0} \in \delta_{p, L}$. Taking this sum separately over the long tiles $\delta_{0, L}$ (resp. short tiles $\delta_{0, S}$ ) we can write $B_{1, L}=x^{\prime}+y^{\prime}$ where $x^{\prime}$ (resp. $y^{\prime}$ ) corresponds to the sum over $\delta_{0, L}$ (resp. $\delta_{0, S}$ ).

Similarly, let $B_{1, S}=B\left(\delta_{p, S}\right)$ where $\delta_{p, S}$ is a short tile of $T_{p}$. Then $B_{1, S}=\sum_{\delta_{0}} \Psi\left(\delta_{0}\right)$ where the sum is over all the $T_{0}$-tiles $\delta_{0} \subset \delta_{p, S}$. Taking this sum separately over the long tiles $\delta_{0, L}$ (resp. short tiles $\delta_{0, S}$ ) we can write $B_{1, S}=u^{\prime}+v^{\prime}$ where $u^{\prime}$ (resp. $v^{\prime}$ ) corresponds to the sum over $\delta_{0, L}$ (resp. $\delta_{0, S}$ ).

Define positive integers:

$$
\begin{aligned}
\alpha_{L} & =\text { number of long } T_{0} \text {-tiles in a long } T_{p} \text {-tile, } \\
\alpha_{S} & =\text { number of short } T_{0} \text {-tiles in a long } T_{p} \text {-tile, } \\
\beta_{L} & =\text { number of long } T_{0} \text {-tiles in a short } T_{p} \text {-tile, } \\
\beta_{S} & =\text { number of short } T_{0} \text {-tiles in a short } T_{p} \text {-tile, } \\
\left|\delta_{n}\right| & =\text { number of } T_{0} \text {-tiles in } \delta_{n} .
\end{aligned}
$$

Let $\delta_{m p, L}$ (resp. $\left.\delta_{m p, S}\right)$ denote a long (resp. short) $T_{m p}$-tile. One then sees that

$$
\begin{aligned}
& \left|\delta_{m p, L}\right|=\alpha_{L} \cdot\left|\delta_{(m-1) p, L}\right|+\alpha_{S} \cdot\left|\delta_{(m-1) p, S}\right|, \\
& \left|\delta_{m p, S}\right|=\beta_{L} \cdot\left|\delta_{(m-1) p, L}\right|+\beta_{S} \cdot\left|\delta_{(m-1) p, S}\right| .
\end{aligned}
$$

By 10.5 .5 on p. 563 of [GS] one knows the following: If $\delta_{n, L}$ (resp. $\delta_{n, S}$ ) is a long (resp. short) $T_{n}$-tile, then the limit of $\left|\delta_{n, L}\right| /\left|\delta_{n, S}\right|$ as $n \rightarrow+\infty$ exists and equals $\tau=(\sqrt{5}+1) / 2$ (in particular nonzero).

With this notation one sees that

$$
b_{m, L}=\frac{\left|\delta_{(m-1) p, L}\right| x^{\prime}}{\left|\delta_{(m-1) p, L}\right| \alpha_{L}+\left|\delta_{(m-1) p, S}\right| \alpha_{S}} b_{(m-1), L}+\frac{\left|\delta_{(m-1) p, S}\right| y^{\prime}}{\left|\delta_{(m-1) p, L}\right| \alpha_{L}+\left|\delta_{(m-1) p, S}\right| \alpha_{S}} b_{(m-1), S}
$$

and

$$
b_{m, S}=\frac{\left|\delta_{(m-1) p, L}\right| u^{\prime}}{\left|\delta_{(m-1) p, L}\right| \beta_{L}+\left|\delta_{(m-1) p, S}\right| \beta_{S}} b_{(m-1), L}+\frac{\left|\delta_{(m-1) p, S}\right| v^{\prime}}{\left|\delta_{(m-1) p, L}\right| \beta_{L}+\left|\delta_{(m-1) p, S}\right| \beta_{S}} b_{(m-1), S} .
$$


Thus we are exactly in the context of part (ii) of Lemma 12 (with a sequence of $2 \times 2$ matrices) applying which the conclusion of Lemma 14 follows.

\subsection{Proof of Theorem 10}

Let $\Psi_{H}: \mathcal{U}_{\delta_{p, L}} \cup \mathcal{U}_{\delta_{p, S}} \rightarrow H$ be a relative $H$-orientation which yields the global $H$ orientation $\Phi_{H}$. Without changing $\Phi_{H}$, we can replace $\Phi_{H}$ by

$$
\Psi_{H}^{(\ell)}: \mathcal{U}_{\delta_{\ell p, L}} \cup \mathcal{U}_{\delta_{\ell p, S}} \rightarrow H
$$

where $\ell$ is a positive integer and $\Psi_{H}^{(\ell)}$ is defined preceding Proposition 7. Choose $\ell$ sufficiently large. Now let $\mathfrak{S}=\mathfrak{S}_{k} \times H$ and let

$$
\Psi_{\mathfrak{S}}=\Psi_{\mathfrak{S}_{k} \times H}: \mathcal{U}_{\delta_{\ell p, L}} \cup \mathcal{U}_{\delta_{\ell p, S}} \rightarrow \mathfrak{S}_{k} \times H
$$

be any map such that

(i) the $H$-coordinate of $\Psi_{\mathfrak{S}_{k} \times H}$ is the same as $\Psi_{H}^{(\ell)}$;

(ii) $\ell$ is large enough so that $\Psi_{\mathfrak{S}_{k} \times H}$ is onto $\mathfrak{S}_{k} \times H$ and in fact the restriction of $\Psi_{\mathfrak{S}_{k} \times H}$ to the subset of long or short $T_{0}$-tiles of both $\mathcal{U}_{\delta_{l p, L}}$ and $\mathcal{U}_{\delta_{l p, S}}$ is onto (see Proposition 7).

Applying Lemma 12 we conclude the following:

(i) For any $n \in \mathbb{N}$ and $s, s^{\prime} \in \mathfrak{S}=\mathfrak{S}_{k} \times H$, let $A_{n, s}$ (resp. $A_{n, s^{\prime}}$ ) be the number of $T_{0}$-tiles $\delta_{0}$ in $\delta_{n l p}$ such that $\Phi_{\mathfrak{S}}\left(\delta_{0}\right)=s$ (resp. $\left.s^{\prime}\right)$. Here $\delta_{n l p}$ is the unique $T_{n l p}$-tile containing a fixed $T_{0}$-tile $\alpha_{0}$ and $\Phi_{\mathfrak{S}}$ is defined as in Definition 5. Then

$$
\lim _{n \rightarrow+\infty} \frac{A_{n, s}}{A_{n, s^{\prime}}}=1
$$

From this it follows that

$$
\lim _{n \rightarrow+\infty}\left(\frac{A_{n, q, h}}{A_{n, q^{\prime}, h^{\prime}}}\right)=1,
$$

where $A_{n, q, h}$ (resp. $\left.A_{n, q^{\prime}, h^{\prime}}\right)$ is the number of $T_{0}$-tiles in $\delta_{n l p}$ of color $q$ (resp. $q^{\prime}$ ) and global $H$-orientation $h$ (resp. $\left.h^{\prime}\right)$. Here $q, q^{\prime} \in\{1, \ldots, k\}$. However, $\left(\mathfrak{S}_{k},\{1, \ldots, k\}\right)$ is the same as $\left(\operatorname{Aut}\left(S^{\prime}\right), S^{\prime}\right)$ ). If a tile in global $H$-orientation $h$ had a color $s^{\prime} \in S^{\prime}$, we assigned to that tile a color from $M$ by taking the $h$-component of $s^{\prime}$. (Recall $S^{\prime}=\prod_{h \in H} S_{h}^{\prime}$, where $S_{h}^{\prime}$ is a union of copies (corresponding to the frequency data) of $G$-orbits in the subsets $M_{h} \subset M$ consisting of allowed colors for tiles in global $H$-orientation $h$.

From this description and from the fact about the limit (as $n \rightarrow+\infty$ ) concluded just before, the assertions (ii) and (iii) of the theorem are evident.

\section{Acknowledgment}

We wish to thank the referee whose numerous suggestions have greatly improved the presentation and organization of this article. 


\section{References}

[GA] Gantmacher, F. R., The Theory of Matrices, vol. II, 1959, translated by K. A. Hirsch, Chelsea, New York (1960).

[GS] Grünbaum, B., and Shephard, G., Tilings and Patterns, Freeman, New York (1987).

[R1] Radin, C., The pinwheel tiling of the plane, Annals of Mathematics, 139 (1994), 661-702.

[R2] Radin, C., Space, tilings and substitutions, Geometriae Dedicata, 55 (1995), 257-264.

Received March 9, 1998, and in revised form August 3, 1998. 